\title{
Comparison of Ultra Capacitors, Hydraulic Accumulators and Batteries Technologies to Optimize Hybrid Vehicle Ecoefficiency
}

\author{
Jonathan Nzisabira, Yannick Louvigny, and Pierre Duysinx \\ LTAS - Automotive Engineering \\ Aerospace and Mechanical Engineering Department \\ University of Liege \\ chemin des chevreuils, 1, B52, 4000 Liege Belgium \\ Email: jnzisabira@student.ulg.ac.be / yannick.louvigny@ulg.ac.be / p.duysinx@ulg.ac.be
}

\begin{abstract}
The main objective of vehicle powertrain hybridization is to simultaneously improve the fuel consumption and environment pollutants impact (Eco-score) without decreasing the vehicle performances and other user satisfaction criteria. Based on a simulation model in ADVISOR, one can evaluate the performances, the emissions and then the Ecoscore and the User Satisfaction for different driving scenarios. To establish a rationale methodology for assessing the eco-efficiency character, we adopt a multidisciplinary optimization approach while minimizing / maximizing both Eco-score and User satisfaction objective functions. The proposed approach is then used to establish a comparison between HEV (hybrid electric vehicle) and HHV (hybrid hydraulic vehicle) configuration by highlighting the effect of different energy storage systems (batteries, ultra capacitors, hydraulic system), mechanical and electric components sizes upon the optimized HEV design. The selected application is the comparison of different mild parallel hybrid buses. Results show that the hybrid electric buses using ultra capacitors have almost the same performances as those using batteries, while the HHV technology can compete with HEV because of the hydraulic components low cost and recyclability if possibly using of water as motor/pump fluid.
\end{abstract}

\section{INTRODUCTION}

Hybrid Electric Vehicles (HEV) are expected to be one of key technologies for future cleaner and fuel efficiency vehicles [1]. Moreover, Hybrid Hydraulic Vehicles (HHV) have recently been the subject of a revival interest with a series of works [2] devoted to the design, the simulation and the optimization of hybrid hydraulic systems for trucks. A success HEV or HHV design requires optimal sizing of its key mechanical, electrical or hydraulic components (See for instance Ref. [2], [3], [4]). In addition, for more hybrid vehicle (HV) efficiency, an optimal management of energy flow (control strategy) is required. Therefore, in their design process, there is a large variety of design variable choices including HV configuration, key mechanical, electrical or hydraulic components sizes and control parameters. Moreover engineers are faced with several conflicting design constraints and objectives aiming at increasing performances and comfort while minimizing environmental impact. We propose here a novel design approach of $\mathrm{HV}$ based on multidisciplinary optimization using genetic algorithms and response surface methods. The approach is based on different coupled analysis problems. At first the HV model is simulated using ADVISOR (advanced vehicle simulator) ([5]). Then emissions can be determined for several driving scenarios and the Ecoscore indicator can be calculated. The User Satisfaction can be evaluated based on performances criteria evaluated from ADVISOR simulations and from simple safety, reliability and daily cost scores, which are computed from simple evaluation tools relying on state-of-the-art of technological information. The goal of this study is to establish a comparison between HEV using Ultra capacitors or Batteries and HHV by highlighting the effect of the energy storage systems, mechanical, electric or hydraulic components sizes (engine, motors) upon the optimized HV design taking care of both Eco-score and User satisfaction for different driving scenarios. Then, the design problem is stated as follows: select mechanical, electric or hydraulic components to minimize the Ecoscore indicator and maximize the user satisfaction criteria subject to discrete valued sizes of components chosen from catalogues. Because of the large number of HV parameters, trial-and-error-based design approaches of a $\mathrm{HV}$ is generally impossible and cumbersome to handle by human intuition. On the contrary, a rationale and efficient design procedure is based on digital simulation and optimization algorithms. As response functions may be noisy and/or discontinuous, derivative-free algorithms are preferred to gradient-based optimization algorithms to solve the problems. Multiobjective versions of Genetic Algorithms are available to handle the ecoefficiency design problem. Finally since response functions are implicit functions of design variables, the numerous direct calls to the simulation code are replaced by surrogate models or metamodels in order to carry out the optimization work with a moderate computational cost. In this study we have selected the software tool Boss Quattro from Samtech [6] to carry out the optimization and the task management tasks of the chain of coupled simulation tools. The approach is illustrated on eco-efficiency design problems of parallel HEV, HHV buses. 


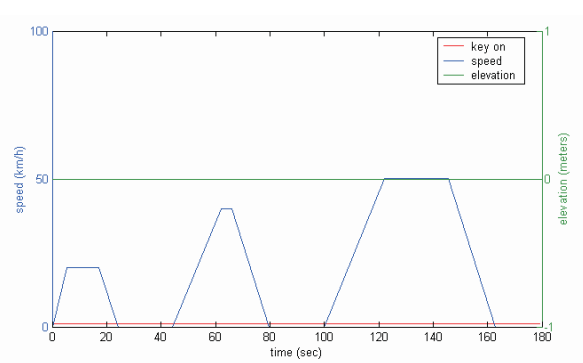

Fig. 1. SORT 2 drive cycle, for easy urban cycle

\section{Simulation}

ADVISOR is used for simulating the fuel consumption, the emissions and the performances of the vehicles. It was initially developed by the National Renewable Energy Laboratory [5] from 1994 to 2002. ADVISOR combines forward /backward facing approach for the vehicle performances simulation. In addition, it offers graphical user interface to select the component modules required to construct the vehicle system. Among several components of a HV, the ICE engine, electric motor and energy storage system are considered as the most critical components. Proper selection of these components mainly affects the vehicle characteristics and performance. To consider the effect of component sizes in the optimization of HV design, ADVISOR approach is to consider a baseline configuration made of selected for the engine and electric motor. The baseline configuration can then scaled up during the design process. For instance for the energy storage system, a battery pack is selected and then the number of battery modules is modified. The baseline scaling factor is naturally considered as the design variables during optimization process. The drive-cycles that had been chosen to simulate and compare the different bus powertrain configurations is SORT2. It is one of the three SORT (standardized on-road test) drive cycles developed by the UITP (International association of public transport) in collaboration with several bus manufacturers. These drive-cycles have been developed in order to provide representative and repeatable tests for European transport public vehicle operators. All of them are synthesis drive cycles based on three trapezoidal speed patterns consisting in an acceleration phase, a plateau representing cruising phase and finally a braking phase followed by an idle period. The size of each phases come from the accumulated background of the UITP. The SORT2 cycle (see figure 1), with a commercial speed of $17 \mathrm{kph}$, mimics an easy urban cycle. The maximum speed is $50 \mathrm{kph}$, the total duration and the traveled distance are bigger.

\section{Simulated BUSSES}

\section{A. Conventional bus}

All buses are based on the Vanhool A300 bus illustrated in figure 2. It is a bus used classically by public transportation company in Belgium with 33 seats and a maximum capacity of 110 persons. It is powered by a $205 \mathrm{~kW}$ diesel Man engine.
TABLE 1: VANHOOL A300 CHARACTERISTICS

\begin{tabular}{|c|c|c|}
\hline Engine & Power & $205 \mathrm{~kW}$ \\
\hline & Max Efficiency & $44 \%$ \\
\hline Aerodynamics & Wet surface & $7.24 \mathrm{~m}^{2}$ \\
\hline & $\mathrm{Cx}$ & 0.79 \\
\hline Tires & Rolling resistance & 0.00938 \\
\hline & Rolling Radius & $0.5 \mathrm{~m}$ \\
\hline
\end{tabular}

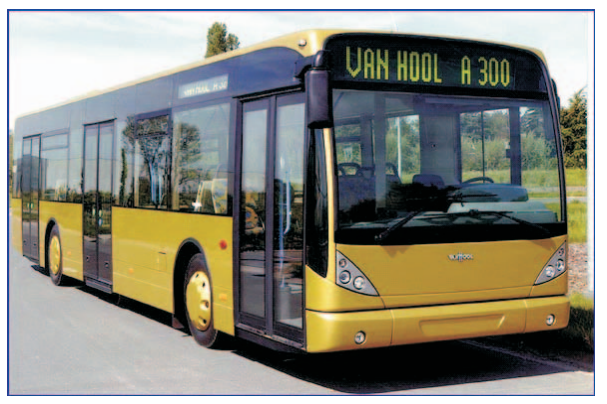

Fig. 2. Bus Vanhool A300

This bus is modeled in ADVISOR using the conventional drivetrain configuration available in the standard library. Taking advantage of ADVISOR library, the bus is modeled using preexisting components for buses or other heavy vehicles with only minor changes. The most important vehicle parameters are given in Table I.

As no information on the original Man engine was available, it was substituted for our study by a $206 \mathrm{~kW}$ diesel engine from Detroit Diesel that is present in the ADVISOR library. As the vehicle mass fluctuates a lot during the day with the number of passengers, an average passengers number is fixed to be 66 .

\section{B. HEV bus with batteries}

The hybrid buses that are modeled and simulated in this study are parallel mild hybrid. This configuration is chosen to be consistent with the architecture of hybrid hydraulic bus and hybrid electric vehicle using super capacitors so that a fair comparison can be conducted. Indeed the parallel architecture is the best configuration to take advantage of the low specific energy of the hydraulic accumulator and low specific energy of the super capacitors. The batteries retained for the simulation are nickel-metal hydride batteries taken from the library. The $\mathrm{NiMH}$ batteries are high energy batteries that are used in standard commercial hybrid vehicle applications (e.g. Toyota Prius and Honda Insight). The chosen powertrain controller is the parallel controller from ADVISOR.

\section{HEV bus with super capacitors}

The super capacitors, whose principles are illustrated in figure 3, are double layer electrochemical capacitors that have a remarkable high capacitance (several hundreds of farads).

Compared to batteries, super capacitors have a higher specific power but they have a smaller specific energy. The super capacitors fill in the gap between capacitors and batteries. The 


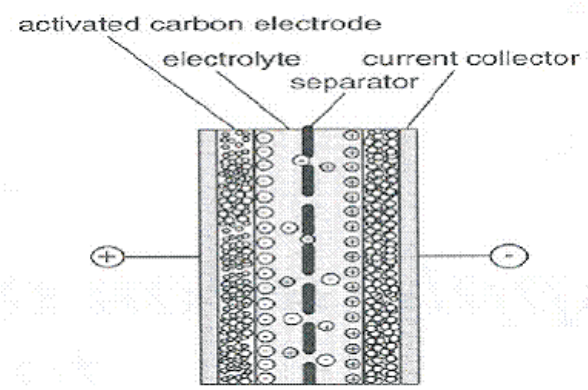

Fig. 3. Double Layer Ultra Capacitor

others great advantages of the super capacitors are their high charge/discharge efficiency and their very long service life and are recyclable without pollutants. The model of HEV bus with super capacitors is very close to the model of HEV bus with batteries. The only important difference comes from the energy storage system characteristics. We chose the energy storage system based on a super capacitor component that is the Maxwell BMOD0018-390V super capacitor module [7].

\section{Hybrid Hydraulic Bus}

In a hybrid hydraulic vehicle, the energy storage system consists of pressurized oil or water. The hydraulic circuit is composed of a low pressure reservoir, a high pressure accumulator and a reversible hydraulic machine operating in two modes, motor and pump. The basic principles of HHV are drawn in figure 4: during braking phases, water or oil is pumped from the reservoir to the high pressure accumulator (the hydraulic machine works as a pump). This energy stored is then used by emptying the accumulator through the hydraulic machine (working as a motor) when needed. There is no model of hydraulic components in ADVISOR. So the hydraulic accumulators and the motor/pump models are derived by developing equivalent (fictitious) electric component models. The storage system is composed of hydraulic accumulators, reservoirs and fluid. In this study, the hydraulic fluid is water because we investigate the applications of a novel design of reversible motor pump [8] that is able to work with water and does not need extra lubricant. The water has two main advantages on other hydraulic fluids such as oil: water has a very low cost and water is clean for the environment in case of leakage. Low pressure reservoir and high pressure accumulator can be found on the market. We selected here reservoir and accumulators for HYDAC catalogue [9].

Hydraulic accumulator models in ADVISOR have been derived from the super capacitor model. The super capacitors are closer, from the specific energy and specific power points of view, to the accumulators than the batteries. In the model, an equivalent number of super capacitors is chosen to obtain the required energy while the mass difference between the two systems is taken into account in the module mass. As the reversible water motor pump is still under development and not fully characterized, we have selected a commercial

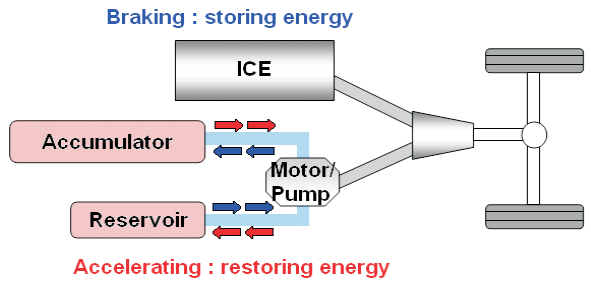

Fig. 4. Basic principle of HHV system

product that has similar performances albeit using oil. The motor/pump is thus chosen among the large range of variable volume piston pumps built by Parker [10]. The pump has also similar efficiency curves than the induction motor present in the ADVISOR library, so that the motor/pump model is tailored from the characteristic curves of an AC induction motor. However its mass has to be modified because the pump is lighter than the equivalent electric motor. One can notice that the HHV configuration is close to the HEV bus with super capacitors. This comes from the similitude in terms of power and energy between the hydraulic system and the super capacitors. The rather low energy density of the HHV technology makes this solution well suited to 'mild' hybrid parallel architecture, where the motor/pump is only used to provide additional power to the crankshaft of the prime mover during short periods of time (vehicle launch) and to recover energy during braking phases. The HHV technology appears to be best suited to heavier vehicles because the surplus of mass is then relatively less important, while the architecture of the chassis offers greater possibility for the packaging of the hydraulic accumulators and reservoirs.

\section{USER SATISFACTION CRITERIA}

Satisfaction of needs is based on several criteria i.e security, daily cost, reliability, performances, etc. Some criteria like performances are easily quantifiable but others like comfort are such qualitative that they can only be estimated by fuzzy description. In this study we will consider only quantitative criteria: cost, performances and security.

1) Performances: Performances include maximal vehicle velocity, acceleration performances and gradeability. They can be evaluated by simulation in ADVISOR by following standard approaches described in classical vehicle theory (see [11] for instance).

2) Security: It is the capability of the vehicle to assure both the passengers and other road users safety. Safety can be based on several criteria like security equipment available on the vehicle, crash test results, static stability factor estimating roll-over resistance [11], etc. As we compare here the same vehicles but with different variants of the propulsion system we can roughly assume that occupant safety is kept the same. But the vehicle mass is the main factor for road security, especially for security of collision partners. Based upon the FARS (Fatal Analysis Reporting System) database, Joksch et al. [12] have estimated the relationship between the mass ratio 
of collision partners, and the fatality ratio of collision partners to be:

$$
\frac{F_{2}}{F_{1}}=\left(\frac{m_{1}}{m_{2}}\right)^{4}
$$

where $m_{1}$ and $m_{2}$ are the mass of vehicle 1 and $2, F_{1}$ and $F_{2}$ are the fatalities in vehicle 1 and 2 . As an example, for a mass ratio of $2: 1$, the formula (1) predicts a fatality ratio of 16:1 between the lighter car and the heavier one. This mean that for vehicle-to-vehicle collisions in which one vehicle weighs twice more than its collision partner, for every fatality unit in the heavier car there would be sixteen in the lighter car. Because of this we decided to focus on the security criteria to evaluate security solely by formula (1) of the mass ratio between the considered vehicle and a reference one. In ADVISOR, the vehicle mass is a function of selected vehicle components.

3) Cost: A simple cost model is introduced to estimate the total vehicle cost which is devised into two costs: An operating cost and an investment cost. The operating cost is related to fuel consumption and maintenance all over the vehicle life. In this study we have assumed a life of 5 years with 500,000 $\mathrm{km}$ which is rather small. The maintenance costs have been neglected because we assume that the maintenance cost is more or less similar for the hybrid and conventional vehicles, which is again a rough approximation. The investment cost is related to key components prices.

4) Aggregated performance criteria: When working with several metaheuristic algorithms such as Genetic Algorithms, one major issue is concerned with considering design constraints. Therefore one strategy to circumvent the problem consists in defining aggregate objective functions or constraints. In order to introduce the performance criteria into the multiobjective approach later, we define here global performance criteria embedding previously defined performance criteria (vehicle maximum velocity, gradeability and the acceleration performance). User satisfaction can therefore be estimated using a linear combination of different criteria weighted by appropriate targets values related to a reference vehicle.

$$
\widetilde{U S}=\frac{\tilde{V}_{\max }}{100}+\frac{20}{\tilde{t}_{a c c}}+\frac{\tilde{p}_{\max }}{6}+\frac{20,000}{\tilde{m}}+\frac{500,000}{\tilde{C}}
$$

Where $\tilde{V}_{\max }$ is the estimated maximum vehicle speed (to be maximized) with $100 \mathrm{kph}$ as target; $\tilde{t}_{a c c}$ is the estimated acceleration time (from 0 to $100 \mathrm{kph}$ ) (to be minimized) with $20 \mathrm{~s}$ as target; $\tilde{p}_{\max }$ is the estimated gradeability (to be maximized) with $6 \%$ as target; $\tilde{m}$ is the vehicle mass (to minimized) with $20,000 \mathrm{~kg}$ as target; $\tilde{C}$ is the total cost estimate (to be minimized). Making use of reference car target criteria also insures the consistency of metric units in the aggregated function. For maximum accuracy, it is a standard procedure in multidisciplinary optimization to estimate each criteria using response surface approximations and then, in a second step, to calculate the user satisfaction from the linear combination of the values coming from the surrogate models.

\section{ECO-SCORE MODEL}

Eco-score [13] is a single environmental indicator which integrates different aspects of the environmental impacts of the road vehicles such as global warming, air quality, energy depletion and noise pollution. The emissions of pollutants considered by Eco-score are related to the direct and indirect emissions. Direct emissions are linked to the use of the vehicle itself (tank-to-wheel) whereas indirect emissions are those related to the extraction and transportation of the raw materials for the fuel production, together with the emissions linked to refining and distributing the carburant (well-to-tank). In this study the direct emissions are obtained by the vehicle simulation in ADVISOR and the indirect emissions are based on the fuel consumption and the indirect emissions factors. The air pollutants cause various damages divided into different categories like global warming, human health impairing effects, harmful effects on ecosystems and building dirtiness. The partial damage of each pollutant is calculated as:

$$
d_{i j}=\beta_{i j} E_{j, \text { total }}
$$

Where $d_{i j}$ is the partial damage of pollutant ' $\mathrm{j}$ ' to category ' $\mathrm{i}$; $\beta_{i j}$ is the impact factor of pollutant ' $\mathrm{j}$ ' to the category ' $\mathrm{i}$; $E_{j, \text { total }}$ is the total contributing emissions of pollutant ' $\mathrm{j}$ ' to the category ' $i$ '. The damages are expressed in common units by category, so the total damage of each damage category can be obtained summing up the partial damages for the different damage categories:

$$
D_{i}=\sum_{j} d_{i j}
$$

In order to quantify the relative severity of the evaluated damages of each damage category, a normalization step based on a specific reference value is performed. The damage associated to the emissions norms EURO IV (directive 98/69/EC) is taken as the reference point.

$$
Q_{i}=\frac{D_{i}}{D_{i, r e f}}
$$

Where $Q_{i}$ is the normalized damage on category ' $\mathrm{i}$ '; $D_{i}^{\prime}$ is the total damage of the assessed vehicle on category 'i'; $D_{i, \text { ref }}$ is the total damage of the reference vehicle on category 'i'. The different damages are weighted before being aggregated to obtain the global damage.

$$
E=W_{i} Q_{i}
$$

Where $W_{i}$ is the weight of damage 'i'.

\section{RESPONSE SURFACE METHOD}

Because of the larger number of function evaluations that can be necessary to carry out optimization process, especially when using meta heuristic algorithms, a standard approach in structural and multidisciplinary optimization consists in resorting to global or local approximation models (see for instance [14], [15]). Approximations will replace direct simulation runs during optimization iterations and will be updated during a limited number of steps ([16]). They provide explicit relations 
TABLE II

OPTIMIZED COMPONENTS SIZES COMPARISON COMPONENTS

\begin{tabular}{|c|c|c|c|}
\hline & PICE & Pmotor & NBat,UCP,HACC \\
\hline CONV.Bus & 205 & & \\
\hline HEV.NiMH & 160 & 100 & 666 \\
\hline HEV.SCAPS & 170 & 86 & 578 \\
\hline HHV & 194 & 88 & 633 \\
\hline
\end{tabular}

TABLE III

OPTIMIZED HV PERFORMANCES COMPARISON

\begin{tabular}{|c|c|c|c|c|}
\hline & ICE & HEV.NiMH & HEV.SC & HHV \\
\hline Fuel $(1 / 100 \mathrm{~km})$ & 62 & 46.5 & 49 & 52 \\
\hline$t_{a c c}(0-64 \mathrm{kph})$ & 20 & 12.0065 & 12.0959 & 12.06 \\
\hline$V_{\max }(\mathrm{km} / \mathrm{h})$ & 106 & 105 & 100.5 & 102.7 \\
\hline$P_{\operatorname{m}} a x$ at $48 \mathrm{kph}$ & 6.5 & 11.3236 & 6.5 & 6.4 \\
\hline $\mathrm{NOX}(\mathrm{g} / \mathrm{km})$ & 132 & 49.0848 & 52.829 & 51.47 \\
\hline $\mathrm{CO} 2(\mathrm{~g} / \mathrm{km})$ & 1618 & 1213.65 & 1278.9 & 1357 \\
\hline $\mathrm{M}(\mathrm{kg})$ & 16242 & 16857 & 16842 & 18721 \\
\hline $\mathrm{C}($ euros$)$ & 451810 & 399161 & 390368 & 374532 \\
\hline Eco score & 1.6 & 1.0598 & 1.1009 & 1.1190 \\
\hline User Satis. US & 6.0 & 8.0 & 7.1 & 6.88 \\
\hline
\end{tabular}

the optimized hybrid buses and the conventional one. For both $\mathrm{HEV}$ and HHV, the engine size is reduced compared to the conventional bus. The fuel consumption and NOX emissions are also reduced in both cases and one can notice subsequently an ECOSCORE improvement. More surprisingly, the performances of $\mathrm{HEV}$ are also improved compared to the conventional bus. Ultimately, in this case, the hybrid electric buses using the batteries have slightly better performances than those one using super capacitors. The HHV performances are inferior to those of HEV because of the excess of mass.

\section{CONCLUSION}

We have carried out a comparison between a conventional bus and a parallel hybrid electric bus using $\mathrm{NiMH}$ batteries or Ultra capacitors (Maxwell BMOD0018-390V model) and a HHV. The eco efficiency comparison is based on a multidisciplinary multiobjective optimization approach of $\mathrm{HEV}$ powertrains accounting for both ECOSCORE for environmental impacts and for an aggregate User satisfaction. The design problem formulation has to optimize the design scaling factors based on the conflicting objective functions of minimizing the environmental impact (ECOSCORE) and maximizing the performance of the vehicle. In this study meta heuristic algorithms such as Genetic Algorithms have been selected in order to cope with noisy and non-smooth response functions. Performance criteria and emissions of vehicles are simulated using the ADVISOR software tool, while the optimization iterative process is carried out in Boss Quattro. In order to reduce the computational cost, one major contribution consists in developing approximations of performance and environmental criteria based on response surface methods. Optimization results show that the hybrid electric bus using super capacitors has almost the same performances as the HEV using NiMH batteries. In the present case of figure, preferred choice would go towards super capacitors because of other appreciable properties compared to the batteries such as higher lifetime, higher efficiency of charge and discharge and no polluting recyclability. The study also showed that HHV technology can provide good fuel saving on city typical drive cycles with frequent stops and starts. The hydraulic storage system has reached an industrial maturity (low cost) and has a very long life time. Thus hybrid hydraulic systems could be an interesting alternative to HEV when considering simultaneously technical and economical considerations. In on-going developments, our approach should be extended to account for more parameters such as the HEV control strategy for further improvement.

\section{ACKNOWLEDGMENT}

Part of this work has been supported by the ENERCARE Project from the Walloon Region of Belgium and by the COST ACTION 542 High Performance Energy Storage for Mobile and Steady State Applications.

\section{REFERENCES}

[1] C. Chan and K. Chau, Modern Electric Vehicle Technology. Oxford Science Publications, 2001.

[2] Z. Filipi, L. Louca, B. Daran, C. Lin, U. Yildir, B. Wu, M. Kokkolaras, D. Assanis, H. Peng, P. Papalambros, J. Stein, D. Szubiel, and R. Chapp, "Combined optimization of design and power management of a hydraulic hybrid propulsion system for 6 x 6 medium truck," Int. J. of Heavy Vehicles Systems, vol. 11, no. 3/4, pp. 372-401, 2004.

[3] J. Garcelon, T. Markel, and K. Wipke, "Hybrid vehicle design optimization," in Proceedings of AIAA/USAF/NASA/ISSMO 8th Symposium on Multidisciplinary Analysis and Optimization, Long Beach, CA, September 6-8, 2000, 2000.

[4] M. Montazeri-Gh and A. Pourmasad, "Appplication of genetic algorithm for simultaneous optimization of hev component sizing and control strategy," Int. J. Alternative Propulsion, vol. 1, no. 1, pp. 63-78, 2006.

[5] T. Markel, A. Booker, T. Hendriks, V. Johnson, F. Kelly, B. Kramer, M. O'Keefe, S. Sprik, and K. Wipke, "Advisor: a system analysis tool for advanced modeling," Journal of Power Sources, vol. 111, no. 2, pp. 255-266, 2002.

[6] Y. Radovcic and A. Remouchamps, "Boss quattro: an open system for parametric design," Structural and Multidisciplinary Optimization, vol. 23, no. 2, pp. 140-152, 2002.

[7] MAXWELL, "http://www.maxwell.com," InternetRessources, 2008.

[8] F. V. Loo, S. Christiaens, J. Nzisabira, P. Mathieu, and P. Duysinx, "Technical and economical study of a new hydraulic motor pump," ENERCARE Project - Technical Report for the Walloon Region of Belgium, 2005, (in French).

[9] HYDAC, "http://www.hydac.com/," InternetRessources, 2008.

[10] PARKER, "http://www.parker.com/," InternetRessources, 2008, parker hydraulic pump/motor division.

[11] T. Gillespie, Fundamentals of vehicle Dynamics. Society of Automotive Engineers, 1992.

[12] H. Joksch, D. Massie, and R. Pichler, "Vehicle aggressivity: Fleet characterization using traffic collision data," Internal Report, The University of Michigan Transportation Research Institute, February 1998.

[13] J. V. Mierlo, "How to define clean vehicles? environmental rating of vehicles," Int. Journal of Automotive Technology, vol. 4, no. 2, pp. 7786, 2003.

[14] W. J. Roux, N. Stander, and R. T. Haftka, "Response surface approximations for structural optimization," International Journal for Numerical Methods in Engineering, vol. 42, no. 3, pp. 517-534, 1998.

[15] R. Jin, W. Chen, and T. Simpson, "Comparative studies of metamodelling techniques under multiple modelling criteria," Structural and Multidisciplinary Optimization, vol. 23, no. 1, pp. 1-13, 2001.

[16] S. David, "Les plans d'expriences et les surfaces de rponse dans bossquattro," Internal Report. SAMTECH S.A, February 1998. 